\section{AB0061 ALTERATIONS OF XANTHINE OXIDOREDUCTASE ACTIVITY IN RED BLOOD CELLS AFTER GLUCOCORTICOID TREATMENT IN RHEUMATOID ARTHRITIS}

E. Mozgovaya ${ }^{1}$, S. Bedina ${ }^{1}$, A. Trofimenko ${ }^{1}$, M. Mamus $^{1}$, S. Spitsina ${ }^{1}$, 1. Zborovskaya ${ }^{1} .{ }^{1}$ Research Institute of Clinical and Experimental Rheumatology named after A.B. Zborovsky, Clinical Biochemistry Lab, Volgograd, Russian Federation

Background: According to modern concepts, rheumatoid arthritis (RA) refers to severe autoimmune rheumatic diseases. The activation of free radical oxidation processes is essential in the development of this disease [1]. Xanthine oxidoreductase is a significant reactive oxygen species source [2]. Despite the great advances in the treatment of rheumatoid arthritis (RA) associated with the introduction of innovative drugs and especially the improvement of the strategy for their use into clinical practice, glucocorticoids still remain an important component of RA pharmacotherapy in actual clinical practice.

Objectives: to evaluate the changes in activities of xanthine oxidoreductase interconvertible forms (xanthine oxidase, EC 1.17.3.2 and xanthine dehydrogenase, EC 1.17.1.4) in lysed red blood cells of RA patients in relation with glucocorticoid treatment.

Methods: 47 RA patients with verified RA and 30 healthy controls were enrolled in the study. The diagnosis was verified using the 2010 ACR/EULAR criteria 2010 All patients have moderate DAS28 scores. RA patients were randomized into 2 groups comparable in gender, age and the principal clinical manifestations Methylprednisolone (Metipred, Orion Corp.), average dose $30 \mathrm{mg} /$ day, and betamethasone (Diprospan, Schering-Plough), single dose7 mg, were administered intramuscularly in the respective groups. Xanthine oxidase (XO) and xanthine dehydrogenase (XDG) activities were measured in lysed red blood cells by spectrophotometric method as previously described [3]. The changes of these enzymes activities were studied in RA patients before and after the injection of glucocorticoids. Statistical comparison tests were selected in according to common guidelines, differences were considered significant when $p<0.05$. Central tendencies were expressed as means \pm SEM.

Results: Mean age of patients in methylprednisolone group was $41.8 \pm 1.05$ years, and mean RA duration $( \pm$ SEM) was $7.9 \pm 0.21$ years. Mean age of patients in diprospan group was $40.9 \pm 1.07$ years, and mean RA duration was $8.0 \pm 0.33$ years. Significant decreases of $X O$ activity and increase of XDG activity were observed in lysed red blood cells of RA patients just after the injection of each glucocorticoid drug. Changes of the enzymatic activities in lysed red blood cells were more pronounced in methylprednisolone group. However enzymatic activity did not reach the level of healthy controls. As described previously, decreased XO activity and increased XDG activity were observed in plasma of RA patients just after the injection of the average therapeutic doses of glucocorticoids, as well as in lysed lymphocytes just after the injection of methylprednisolone [4].

Conclusion: Treatment with methylprednisolone and betamethasone can affect the balance of XO/XDG activity and increase the antioxidant potential of the blood. This effect can exert beneficial influence on autoimmune inflammation in RA.

REFERENCES:

[1] Mateen S., et al. Increased reactive oxygen species formation and oxidative stress in rheumatoid arthritis. PLoS ONE 2016;11(4):e0152925.

[2] Çimen M.Y., et al. Oxidant/antioxidant status of the erythrocytes from patients with rheumatoid arthritis. Clin Rheumatol 2000;19(4):275-277.

[3] Zborovskaya I.A., et al. Influence of analgetics on plasma and lymphocytic activity of the purine metabolism enzymes in rheumatoid arthritis patients. Russian Journal of Pain 2018;3:47.

[4] Mozgovaya E.E., et al. Xanthinoxidase and xanthine dehydrogenase activities in rheumatoid arthritis after glucocorticoid treatment. Osteoporosis International 2019;30(2):S433-434

Disclosure of Interests: None declared

DOI: 10.1136/annrheumdis-2021-eular.3168

\section{$\mathrm{AB} 0062$ \\ TIME COURSE OF INTESTINAL PERMEABILITY AND BACTERIAL TRANSLOCATION IN THE MODEL OF ADJUVANT-INDUCED ARTHRITIS}

S. Hecquet ${ }^{1}$, P. Totoson ${ }^{2}$, H. Martin ${ }^{2}$, C. Peyronnel ${ }^{2}$, M. Tournier ${ }^{2}$, P. Saas ${ }^{3}$, J. P. Pais-De-Barros ${ }^{4}$, D. Wendling ${ }^{1}$, C. Demougeot ${ }^{2}$, F. Verhoeven ${ }^{1} .{ }^{1}$ Centre hospitalier régional universitaire de Besançon, Rheumatology, Besançon, France; ${ }^{2}$ EA4267 PEPITE LAB, Physiology, Besançon, France; ${ }^{3}$ UMR 1098 , Interactions Hôte Greffon Tumeur, Besançon, France; ${ }^{4}$ UMR 1231, Plateforme de lipidomique, Dijon, France

Background: Intestinal inflammation, dysbiosis, intestinal permeability (IP) and bacterial translocation (BT) have been identified in patients with spondyloarthritis but the time at which they appear and their contribution to the pathogenesis of the disease is still a matter of debate.

Objectives: To investigate the time-course of intestinal inflammation, IP and $\mathrm{BT}$ in a rat model of reactive arthritis, a subgroup of $\mathrm{SpA}$, the adjuvant-induced arthritis model.

Methods: Adjuvant-induced arthritis (AIA) was induced in 6-week-old male Lewis rats by an injection at the base of the tail of Mycobacterium butyricum with incomplete Freund's adjuvant (Day (D) 0 ). Control rats received saline using the same procedure. Body weights and a clinical arthritis score were daily assessed. A group of AIA and control rats ( $n=15$ per group) were euthanized at three different times of arthritis: D4 for the pre-arthritic phase (AIA-preclinical), D11 for the onset of arthritis (AIA-onset) and D28 for the acute phase (AIA-acute). In each group (AIA and control, $n=15$ per group)), IP was assessed by measuring plasma levels of zonulin (ELISA) and ileal mRNA expression of zonulin and occludin (RT-qPCR), BT was studied by measuring bacterial endotoxins (or LPS, by LCMS $^{2}$ method), soluble CD-14 (sCD14, ELISA) and ileal mRNA expression of TLR-4, and intestinal inflammation was assessed by measuring ileal mRNA expression of IL-8, IL-33, IL-17, IL-23p19 and TNF- $\alpha$ (RT-qPCR). Joint damage was assessed by the determination of a clinical and radiographic score of hind paws.

Results: Body weights of AIA rats decreased from D4 to D28 as compared to controls, in parallel to the development of a severe clinical and radiographic arthritic disease from D11 and D28. Compared to control rats, AIA induced an increase in plasma zonulin levels at D4, D11 but not at D28. Ileal mRNA zonulin overexpression occurred at D11 while occludin was unchanged. As early as Day 4 (preclinical phase), mRNA of IL-8, IL-33 and IL-17 were overexpressed in ileum from AIA. At Day 11 (onset), overexpression of IL-8 persisted and mRNA of TNF- $\alpha$ and IL-23p19 increased in AIA. Neither LPS levels nor ileal mRNA expression of TLR-4 were changed by arthritis whatever the phase of arthritis. By contrast, blood levels of SCD-14 was significantly increased in the AIA group at all stages of arthritis. No correlation was found between clinical and radiographic arthritis scores and zonulin or LPS levels. Conversely, a negative correlation was observed between intestinal IL-8 mRNA expression and arthritis score $(r=-0.3, p=0.02)$.

Conclusion: In an animal model of SpA, intestinal inflammation and increased intestinal permeability occur prior to joint inflammation, suggesting a role of these disorders in the pathogenesis of this disease.

Acknowledgements: I would like to thank the Société Française de Rhumatologie for its support in this work.

Disclosure of Interests: None declared

DOI: 10.1136/annrheumdis-2021-eular.3207

\section{AB0063 DIFFICULT-TO-TREAT RHEUMATOID ARTHRITIS: A BIOMARKER SCREENING PILOT STUDY}

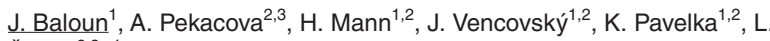
Šenolt ${ }^{2,3}{ }^{1}$ Institute of Rheumatology, Department of Rheumatology, Prague, Czech Republic; ${ }^{2} 1$ st Faculty of Medicine, Charles University, Department of Rheumatology, Prague, Czech Republic; ${ }^{3}$ Institute of Rheumatology, Department of Experimental Rheumatology, Prague, Czech Republic

Background: Despite modern therapeutic approaches, many patients with rheumatoid arthritis (RA) remain symptomatic after several cycles of treatment and may become so called Difficult-To-Treat (D2T)1. D2T RA is a multifactorial condition in which different factors may be major determinants of the persistence of signs and symptoms, which is seldom caused by drug resistance only2. Discovering new biomarkers is necessary to develop tailored therapies that will be effective in an individual patient at each stage of the disease.

Objectives: The primary aim of this pilot study was to validate a target proteomic technique for the proteome profiling of the two cohorts of RA patients and controls. Moreover, we searched for potential plasma biomarker(s) predicting D2T RA.

Methods: Seven RA patients with persistent remission on biological therapy in two consecutive examinations 12 wks apart (mean age $59.6 \pm 14$ yrs), seven D2T RA patients fulfilling proposed EULAR definition of D2T RA1 (mean age $59.3 \pm 13$ yrs), and six healthy controls (mean age $58.8 \pm 15 \mathrm{yrs}$ ) were included in this study. All subjects were females and their samples were collected before starting biological therapy. We employed Thermo Orbitrap Fusion paired with nano-flow UHPLC Dionex Ultimate 3000. Prior to quantification, 125 plasma proteins were modified by Peptiquant Plus Human kit to increase the sensitivity. Data were analysed by ANOVA and Tukey`s posthoc test with false-discovery-rate adjustment. Results: The target proteome profiling reliably quantified 92 from 125 labelled proteins. Our follow-up statistical analysis revealed ten plasma proteins, which significantly differed among groups. Notably, we found significantly different plasma levels of paraoxonase/arylesterase 1 (PON1), an esterase with an antioxidant characteristic preventing lipid peroxidation ${ }^{3}$, between RA patients and controls and between RA patients with persistent remission and D2T RA patients. 
Conclusion: Using target proteome profiling technique, we demonstrated PON1 as a potential biomarker of D2T RA. However, these results have to be validated on a larger cohort.

\section{REFERENCES:}

[1] Nagy G, Roodenrijs NMT, Welsing PMJ, et al. EULAR definition of difficult-To-Treat rheumatoid arthritis. Annals of the Rheumatic Diseases 2021;80:31-5.

[2] de Hair MJH, Jacobs JWG, Schoneveld JLM, van Laar JM. Difficult-to-treat rheumatoid arthritis: an area of unmet clinical need. Rheumatology (Oxford, England) 2018 Jul 1;57(7):1135-1144

[3] Isik A, Koca SS, Ustundag B, Celik H, Yildirim A. Paraoxonase and arylesterase levels in rheumatoid arthritis. Clinical Rheumatology 2007;26:342-8.

Acknowledgements: This research was supported by MHCR No. 023728 and authors thank Proteomics Service Laboratory, Faculty of Science, Charles University for performing the LC-MS/MS analysis.

Disclosure of Interests: None declared

DOI: 10.1136/annrheumdis-2021-eular.3290

\section{AB0064 EVALUATION OF QUALITY OF LIFE IN RHEUMATOID ARTHRITIS USING SF-36 AND HAQ SCALES}

M. Brahem ${ }^{1}$, M. Hassayoun ${ }^{1,1}$, H. Hachfi ${ }^{1}$, R. Sarraj ${ }^{1}$, M. Ardhaoui ${ }^{1}$, A. Ben Salem $^{1}$, S. Abdellatif ${ }^{1}$, R. Ben Tekaya ${ }^{1}$, Y. Mohamed ${ }^{1}{ }^{1}$ Taher Sfar Hospital, Rheumatology, Mahdia, Tunisia

Background: The assessment of health-related quality-of-life (HRQoL) in rheumatoid arthritis (RA) is becoming a common tool in clinical practice. The medical outcomes survey short form 36 (SF- 36) is one of the most widely used tools for measuring $H R Q \circ L$ in RA as well as the $H A Q$ scale.

Objectives: The aim of our study is to evaluate the impact of the RA in the quality of life (QoL) of our patients using the SF-36 and the HAQ questionnaires.

Methods: This is a cross-sectional study during a period of the year 2020, including 70 patients followed in the department of Rheumatology in Mahdia, Tunisia. All patients were diagnosed with RA based in ACR 1987/EULAR 2010. We evaluated for each patient, the mean global scale and the eight domains of SF-36 (physical functioning (PF), role physical (RP), bodily pain (BP), general health (GH), vitality (VT), a social functioning (SF), role emotional (RE) and mental health $(\mathrm{MH})$ ), scored from 0 (worst) to 100 (best).

Results: Our study included 70 patients (59 females/11males) with an age ranged from 21 to 76 years. The mean age was $54 \pm 12$ years. The mean duration of the disease was $11 \pm 10$ years [1-40]. The mean number of tender joints was $9.7 \pm 9.4$ and swollen joints were $4.2 \pm 6$.1. The mean disease activity score (DAS28) was $4.6 \pm 1.9$ [1.2-8.4]. The mean HAQ score was $1.5 \pm 1.3,47.1 \%$ of patients had specific joint deformations, $82.9 \%$ had radiologic involvement and $31.4 \%$ had osteoporosis. The biologic analysis showed that the mean ESR was $46.7 \pm 30.5$ and the CRP was $15.8 \pm 23.3$. Rheumatoid factors were positive in $42.9 \%$ of cases, the ACPA were positive in $50 \%$ of cases. $84.3 \%$ of RA patients were treated by methotrexate, $4.3 \%$ were treated by salazopyrin and $11.4 \%$ were treated by biologic treatments.

The SF-36 global score was $50.4 \pm 26.3$ [15.3-92.8]. 46 patients $(65.7 \%$ of cases) had impaired QoL (SF-36<66.7). The means of different domains (PF, RP, BP, GH, VT, SF, RE, MH) were respectively $51 ; 41.4 ; 51.4 ; 50 ; 51.2 ; 57.7 ; 41.9 ; 59.2$. The most severely impacted domains were the RP and RE.

Our study showed a significant correlation between the SF-36 global score and the number of tender joints $(p=0.002)$, the DAS28 $(p=0.017)$ and the $\mathrm{HAQ}(\mathrm{p}=0.000)$

Conclusion: Our study showed that $65.7 \%$ of RA patients presented impaired QoL (SF-36<66.7), which is associated with high disease activity. So it's important to jugulate the disease, in order to ameliorate the quality of life of our patients. REFERENCES:

[1] Matcham, F., Scott, IC, Rayner, L., Hotopf, M., Kingsley, GH, Norton, S.,.. Steer, S. (2014). L'impact de la polyarthrite rhumatoïde sur la qualité de vie évalué à l'aide du SF-36: une revue systématique et une méta-analyse. Séminaires sur l'arthrite et les rhumatismes, 44 (2), 123-130. doi: 10.1016 / j.semarthrit.2014.05.001.

Disclosure of Interests: None declared

DOI: 10.1136/annrheumdis-2021-eular.3309

\section{AB0065 HGF/C-MET SIGNALING PROMOTE ANGIOGENESIS THROUGH CXCL16 IN RHEUMATOID ARTHRITIS}

M. Hosonuma ${ }^{1,2,3,4}$, T. Isozaki ${ }^{2}$, H. Furuya ${ }^{2}$, Y. Yamazaki ${ }^{5}$, Y. Ikari ${ }^{2}$, S. Nishimi ${ }^{2}$, S. Ishii ${ }^{2}$, A. Maeoka ${ }^{2}$, T. Tokunaga ${ }^{2}$, K. Wakabayashi ${ }^{2}$, N. Konishi $^{2}$, S. Fukuse $^{2}$ K. Ishikawa ${ }^{6}$, N. Sakai ${ }^{7}$, K. Inagaki ${ }^{6}$, T. Kasama ${ }^{2} .{ }^{1}$ Clinical Research Institute for Clinical Pharmacology \& Therapeutics, Showa University, Department of Clinical Immuno Oncology, Tokyo, Japan; ${ }^{2}$ Showa University, Division of Rheumatology, Department of Medicine, Tokyo, Japan; ${ }^{3}$ Showa University,
Division of Medical Oncology, Department of Medicine, Tokyo, Japan; ${ }^{4}$ Showa University, Department of Pharmacology, School of Medicine, Tokyo, Japan;

${ }^{5}$ Showa University School of Pharmacy, Division of Toxicology, Department of Pharmacology, Toxicology and Therapeutics, Tokyo, Japan; ${ }^{6}$ Showa University School of Medicine, Department of Orthopaedic Surgery, Tokyo, Japan;

${ }^{7}$ Showa University, Department of Pharmacology, School of Dentistry, Tokyo, Japan

Background: Hepatocyte growth factor (HGF) binds to the receptor tyrosine kinase c-Met and is a multifunctional cytokine that promotes processes such as cell proliferation, survival, differentiation, migration and angiogenesis [1]. We previously reported that HGF is produced by inflammation in the RA synovium, and activates monocyte migration to the synovium and promotes bone destruction through its own chemotactic effect and enhanced chemokine production in the synovium [2]

Objectives: Therefore, we next aimed to determine the role of HGF in RA angiogenesis.

Methods: The expression of HGF / c-Met in the serum and synovial tissues (STs) of RA patients and controls and human umbilical vein endothelial cells (HUVECs) was evaluated by ELISA and immunostaining. The effect of HGF/cMet signaling on the promotion of CXCL16 production from HUVECs and RA fibroblast-like synoviocytes (FLSs) was determined by ELISA. To examine the role of HGF in angiogenesis, we performed in vitro Matrigel assays using HUVECs treated with HGF.

Results: HGF in serum in treatment-naive RA patients was significantly higher than that in controls and HGF in serum in treatment-resistant RA showed a sig nificant positive correlation with CXCL16. c-Met were expressed on vascular endothelial cells of RA STs and HUVECs. Stimulation of HUVECs with HGF dose-dependently increased CXCL16 production. c-Met signal inhibition by SU11274 suppressed TNF- $\alpha$ stimulation-enhanced CXCL16 production by RA FLSs in a dose-dependent manner. Furthermore, HGF induced HUVEC tube formation by 1.8 -fold.

Conclusion: HGF is produced by inflammation in the RA synovium, and activates angiogenesis through its own potent angiogenic effect and enhanced production of CXCL16 in the synovium. These results indicate that a strategy targeting c-Met signalling may be important for resolving treatment-resistant RA.

\section{REFERENCES:}

[1] Nakamura T, Nishizawa T, Hagiya M, et al. Molecular cloning and expression of human hepatocyte growth factor. Nature. 1989 Nov 23;342(6248):440-3

[2] Hosonuma M, Sakai N, Furuya $\mathrm{H}$, et al. Inhibition of hepatocyte growth factor/c-Met signalling abrogates joint destruction by suppressing monocyte migration in rheumatoid arthritis. Rheumatology (Oxford). 2021 Jan 5:60(1):408-419

Disclosure of Interests: None declared

DOI: 10.1136/annrheumdis-2021-eular.3491

\section{AB0066 MECHANISMS OF TOTAL SAPONINS OF PANAX JAPONICUS MITIGATES COLLAGEN-INDUCED ARTHRITIS}

Z. Feng ${ }^{1}$, X. Guo ${ }^{1}, \mathrm{~J} \mathrm{Ji}^{1}, \mathrm{X} . \mathrm{Hou}^{2}, \mathrm{Y} . \mathrm{Luo}^{1}, \mathrm{X} . \mathrm{Fu}^{1} .{ }^{1}$ Medical College of China Three Gorges University, Third-Grade Pharmacological Laboratory on Chinese Medicine Approved by State Administration of Traditional Chinese Medicine, Yichang, China; ${ }^{2}$ Medical College of China Three Gorges University, Institute of Rheumatology, The First College of Clinical Medical Sciences, Yichang, China

Background: Total saponins of Panax japonicus (TSPJ) are extracted from Panax japonicus (T.Nees) C.A.Mey and have achieved a good therapeutic effect in the treatment of rheumatoid arthritis (RA). Unfortunately, the mechanism of TSPJ acting on RA is not clear.

Objectives: To investigate the potential mechanisms and key targets of TSPJ on RA.

Methods: The raw data were downloaded from the Gene Expression Omnibus (GEO) database, and the RStudio3.6.1 software was used to identify differentially expressed genes (DEGs). The potential targets of active compounds from TSPJ were predicted by the Pharmmapper and SwissTargetPrediction databases. Based on the overlapping genes, we used Cytoscape 3.7.2 software to construct a protein-protein interactions (PPI) network and to determine the mechanisms of the treatment by Kyoto Encyclopedia of Genes and Genomes (KEGG) analysis. Collagen-induced arthritis (CIA) model was established and treated with different doses of TSPJ. Arthritis index (AI) and histology score were used to evaluate the symptoms of $\mathrm{CIA}$. The levels of vascular endothelial growth factor (VEGF), hypoxia-inducible factor 1 (HIF-1), IL-1 $\beta$, and IL-17A tested by enzyme linked immunosorbent assay and real time-quantitative Polymerase Chain Reaction. 